\title{
Feedback by supermassive black holes in galaxy evolution: impacts of accretion and outflows on the star formation rate
}

\author{
Mojtaba Raouf, ${ }^{1,2 \star}$ Joseph Silk, ${ }^{3,4,5 \star}$ Stanislav S. Shabala ${ }^{\circledR}, 6,7$ Gary A. Mamon ${ }^{\circledR}, 3$ \\ Darren J. Croton, ${ }^{8}$ Habib G. Khosroshahi ${ }^{1,3}$ and Ricarda S. Beckmann ${ }^{\oplus 3}$ \\ ${ }^{1}$ School of Astronomy, Institute for Research in Fundamental Sciences (IPM), Tehran, 19395-5531, Iran \\ ${ }^{2}$ Korea Astronomy and Space Science Institute, 776 Daedeokdae-ro, Yuseong-gu, Daejeon 305-348, Korea \\ ${ }^{3}$ Institut d'Astrophysique de Paris (UMR 7095: CNRS \& Sorbonne Université), 98 bis Bd. Arago, F-75014 Paris, France \\ ${ }^{4}$ Department of Physics and Astronomy, Johns Hopkins University, Baltimore, MD 21218, USA \\ ${ }^{5}$ BIPAC, Department of Physics, University of Oxford, Keble Road, Oxford OX1 3RH, UK \\ ${ }^{6}$ School of Natural Sciences, Private Bag 37, University of Tasmania, Hobart, TAS 7001, Australia \\ ${ }^{7}$ Centre for Astrophysics Research, School of Physics, Astronomy and Mathematics, University of Hertfordshire, College Lane, Hatfield, Hertfordshire AL10 \\ $9 A B, U K$ \\ ${ }^{8}$ Centre for Astrophysics \& Supercomputing, Swinburne University of Technology, PO Box 218, Hawthorn, Victoria 3122, Australia
}

Accepted 2019 March 22. Received 2019 March 12; in original form 2018 November 13

\begin{abstract}
We describe a physical model of the outflows produced as a result of gas accretion on to a black hole, and the resultant changes to star formation rates and efficiencies in galaxies, using the RADIO-SAGE semi-analytic galaxy formation model. We show that the ratio of outflow rate to SFR of galaxies is mainly driven by black hole mass and virial halo mass, and show that the SFR is higher than the outflow rate at low black hole masses. The model consistently reproduces the observed evolution of star formation rate density from $z=6$ to $z=0$, as well as the trend of the stellar mass - halo mass relations. We show the characteristic growth of massive galaxies influenced by AGN feedback at different redshifts. We find feedback to be prevalent in the most massive galaxy haloes, inhibiting the cooling catastrophe.
\end{abstract}

Key words: galaxies: active-galaxies: evolution-galaxies: formation-galaxies: generalgalaxies: haloes - galaxies: star formation.

\section{INTRODUCTION}

One of the major goals of modern astronomy is to understand the star formation history of the Universe. At the present epoch, the cosmic star formation rate (SFR) density increases to $z \simeq 1$ (e.g. Hopkins \& Beacom 2006; Villar et al. 2008; Sobral et al. 2009) then reaches a peak around $z \simeq 2$ and decreases at higher redshifts (Bouwens et al. 2008; Pérez-González et al. 2008). There have been extensive efforts to understand the evolution of galaxy SFRs through the relatively tight correlation between galaxy SFR and stellar mass known as the galaxy SFR main sequence, with a shallower slope at lower redshift (Elbaz et al. 2007; Daddi et al. 2007; Salim et al. 2007; addi et al. 2009; Stark et al. 2009; Gonzalez et al. 2009; Oliver et al. 2010; Pearson et al. 2018). Furthermore, the star formation main sequence galaxies show much longer typical depletion times with respect to the entire mass range of star-forming galaxies via studies of the dust and gas content of $z \sim 2$ galaxies (Magdis et al.

^E-mail: m.raouf@ipm.ir (MR); silk@iap.fr (JS) 2012b; Béthermin et al. 2015; Genzel et al. 2015; Tacconi et al. 2018).

Every massive galaxy could host a supermassive black hole $(\mathrm{SMBH})$ at the centre. This provides a mechanism for explaining its presence of Active Galactic Nuclei (AGN), which suppress excessive star formation and hence galaxy growth (Silk \& Rees 1998). A principal motivation to include AGN feedback in galaxy formation models is to understand how the suppression of rapid gas cooling then leads to a suppression of the formation of new stars. In the most massive haloes, the ratio of galaxy stellar mass to dark matter halo mass decreases with increasing mass (Behroozi et al. 2013a), rendering supernova feedback inefficient (Dubois \& Teyssier 2008; Dashyan et al. 2018). AGN feedback could provide an effective method for quenching massive galaxies, as well as regulating the growth of supermassive black holes (SMBH) (Binney \& Tabor 1995; Benson et al. 2003; Di Matteo, Springel \& Hernquist 2005; Bower et al. 2006; Croton et al. 2006; Sijacki et al. 2007; Cattaneo et al. 2009; Fabian 2012).

The gas content of the galaxy could directly be influenced by feedback from the AGN jets, which heat up the surrounding gas 


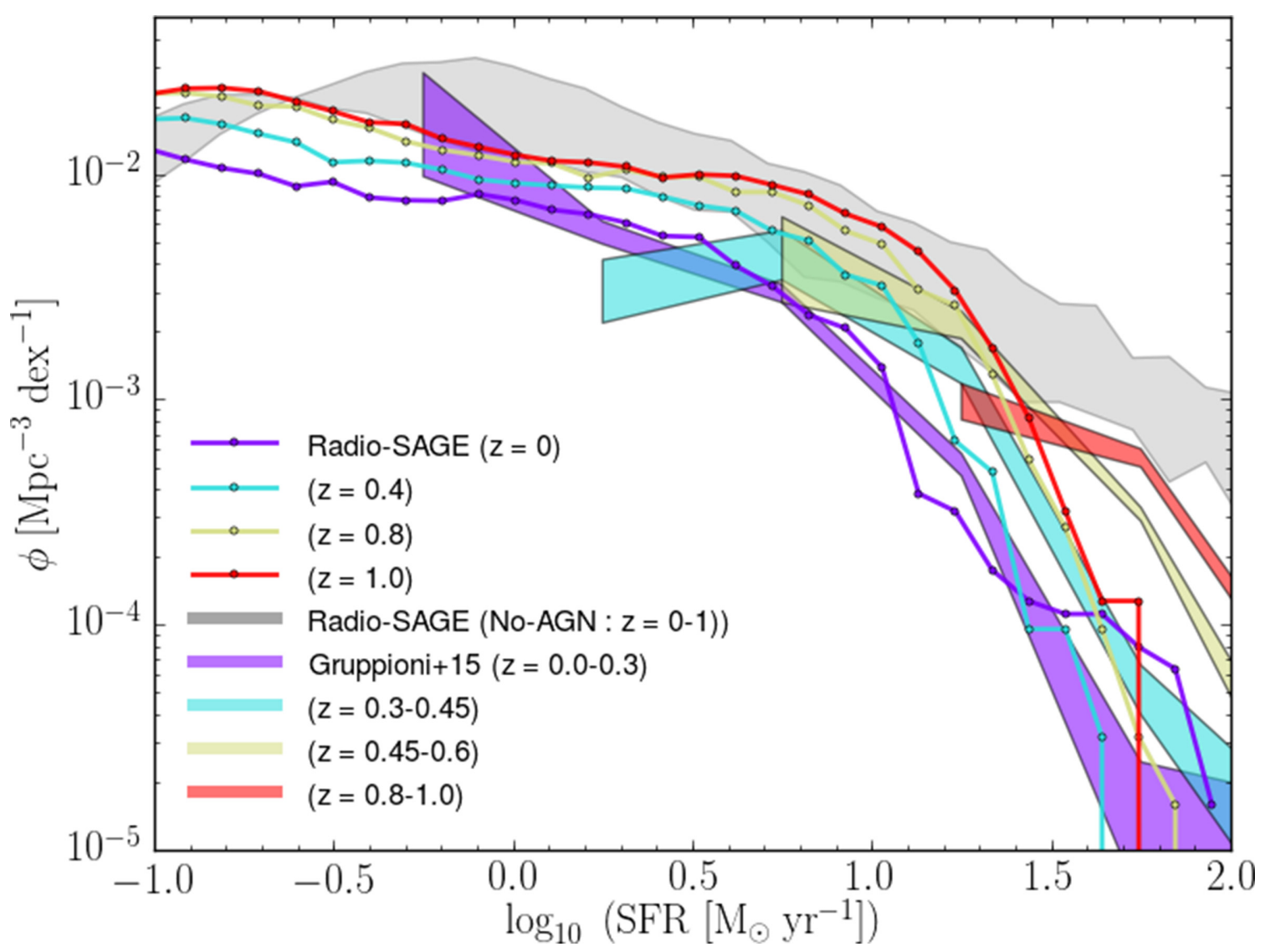

Figure 1. Distribution of galaxy star formation rates in different redshift bins from $z=0$ to 1 . Solid lines show Radio-SAGE predictions. Shaded curves are observations by Gruppioni et al. (2015) at $0<z<1$. The grey-shaded region shows the model without AGN feedback for the full-redshift range.

leading to negative feedback on star formation. ${ }^{1}$ At higher redshift, the powerful energy-driven winds from quasars could lead to a rapid decline of the star formation rate (Farrah et al. 2012; Maiolino et al. 2012; Page et al. 2012; Cicone et al. 2014; Costa, Sijacki \& Haehnelt 2015; Williams et al. 2016). Observationally, there are correlations between the properties of supermassive black holes and host galaxies in the different modes of accretion power (Ferrarese \& Merritt 2000; Gebhardt et al. 2000; Di Matteo et al. 2008; Booth \& Schaye 2009; Dubois et al. 2012; Sijacki et al. 2015; Volonteri et al. 2016). Observational evidence shows that there are significant outflows in massive galaxies using only $5-10$ per cent of accretion power (Moe et al. 2009; Saez, Chartas \& Brandt 2009; Dunn et al. 2010) including highly uncertain measurements with many assumptions going into efficiency estimates, and in part due to different AGN phenomena other than the assumptions of the jet model. By using a larger fraction of accretion power, the RADIOSAGE model provides the necessary energy input to both quench star formation and to avoid the overcooling problem, and reproduces both the observed optical and radio luminosity functions at the present epoch (Raouf et al. 2017).

Black hole growth strongly depends on its environment. For instance, Raouf et al. (2016) using hydrodynamical simulations show that the black holes hosted by the brightest group galaxies

${ }^{1} \mathrm{AGN}$ feedback might even accelerate star formation by further compressing the cold gas of the galaxy, in a so-called positive feedback mode (Silk 2005; Crockett et al. 2012; Gaibler et al. 2012; Santini et al. 2012; Bieri et al 2015) in dynamically young groups are more massive than those with a similar stellar mass but residing in dynamically old groups (for a definition of old and young groups, see Raouf et al. 2014) resulting in a strong dependency of the black hole growth on its environment. They also showed that such old groups with massive black holes have a lower rate of black hole accretion in comparison to the young systems, in agreement with observations and semi-analytic model predictions for radio luminosity (Khosroshahi et al. 2017; Raouf et al. 2018).

The underlying physics of black hole accretion, and especially the mechanisms connecting the accretion flow with large-scale outflows, are still outstanding problems in astrophysics. Here, we address the following questions, How does accretion of the SMBH affect the SFR? and what is the connection between SFR and AGN outflow rate in the galaxies hosted by a massive black hole? These are debated in a number of publications (e.g. Quintero et al. 2004; Thomas et al. 2005; Schawinski et al. 2014; Kaviraj et al. 2015; Shabala et al. 2017). The rest of the paper will focus on the relationship between supermassive black hole growth and outflows. This paper is organized as follows: In Section 2, we describe our Nbody and RADIO-SAGE model framework with publicly available code as a sub-group of the original SAGE repository. ${ }^{2}$ In Section 3, we describe the constraints on our model. Results and predictions are discussed in Section 4. We present the summary of our results in Section 5.

\footnotetext{
${ }^{2}$ https://github.com/mojtabaraouf/sage
} 


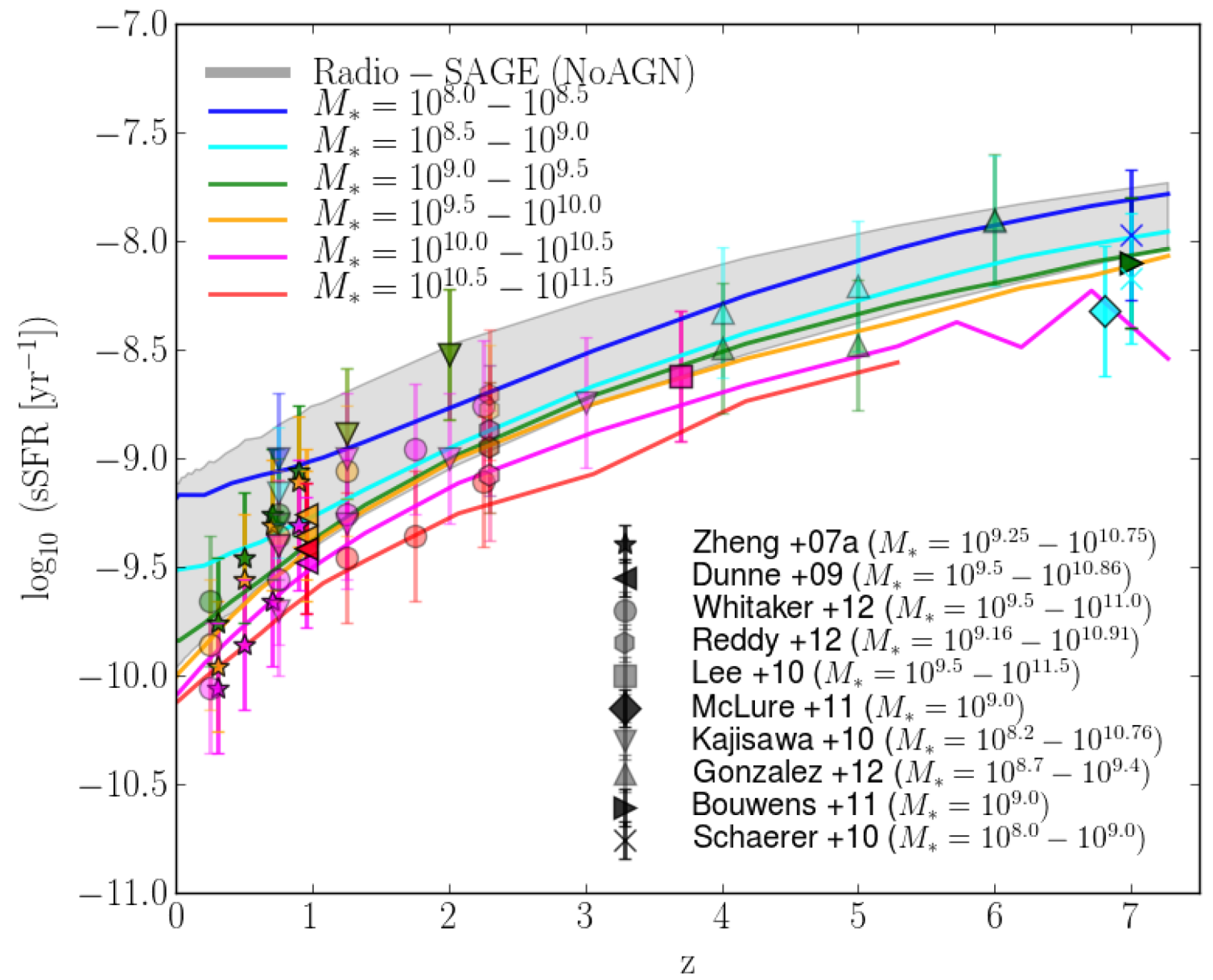

Figure 2. Redshift evolution of the median specific star formation rate from $z=0$ to 7 , for different stellar mass bins. The observed evolution is indicated by symbols (including, Zheng et al. 2007; Dunne et al. 2009; Whitaker et al. 2012; Reddy et al. 2012; Lee et al. 2010; McLure et al. 2011; Kajisawa et al. 2010; Gonzalez et al. 2012; Bouwens et al. 2011 and Schaerer \& de Barros 2010), where the colour of each symbol denotes the relevant stellar mass range. The vertical error bars indicate the estimated intrinsic scatter in SSFR. The grey-shaded region shows the model without AGN feedback for all masses from RADIO-SAGE.

\section{THE GALAXY FORMATION MODEL; RADIO-SAGE}

We only give a brief introduction to the base RADIO-SAGE galaxy formation model here and refer the interested reader to Raouf et al. (2017) and the original SAGE paper (Croton et al. 2016) for a full description. We use the Millennium Simulation (Springel et al. 2005) N-body dark matter halo merger trees as input into RADIOSAGE, which has been updated with a new gas density profile and AGN physics calibrated to match key observations, as explained below.

The Millennium Simulation, containing $2160^{3}$ particles of mass $8.6 \times 10^{8} h^{-1} \mathrm{M}_{\odot}$ within the box volume of $\left(500 h^{-1} \mathrm{Mpc}\right)^{3}$, was run using the popular GADGET-2 code and adopted a cosmological model consistent with the first year Wilkinson Microwave Anisotropy Probe data (WMAP-1, with parameters $\Omega_{\mathrm{m}}=0.25$, $\Omega_{\Lambda}=0.75$ and $H_{0}=100 h \mathrm{~km} \mathrm{~s}^{-1} \mathrm{Mpc}^{-1}$, where $h=0.73$ Spergel et al. 2003). The simulation covers redshift $z=127$ to the present epoch and stores its data in 64 separate snapshots. The SUBFIND algorithm (Springel et al. 2001) was then applied to the Friendsof-Friends (FOF; Davis 1985) catalogue to identify subhalos by restricting the boundaries of substructures. The halo mass resolution is 20 particles, while halo merger trees are provided by the LHALOTREE.
Following our previous study to ensure RADIO-SAGE produces a galaxy population akin to that observed around us, it is calibrated to statistically match a set of key observables up to $z=7$, with a main focus on star formation. It uses a more realistic hydrostatic hot gas density profile to calculate cooling, the AGN jet can heat the hot gas to higher temperatures than the original SAGE, and the cooling rate is affected by both this new temperature and the jet-inflated cavities.

\subsection{Cooling and black hole accretion}

In this model, baryons ${ }^{3}$ initially form as diffuse hot gas with primordial composition around the galaxy. We adopted the density profile described by Makino et al. (1998), which closely matches the observationally fit $\beta$-model but with a robust theoretical foundation (Yates et al. 2018).

Supermassive black holes grow by merging and accretion of gas during major mergers of galaxies as same as the original SAGE

${ }^{3}$ Here, the mass fraction in baryons associated with every dark matter halo is taken to be $f_{b}=0.17$, consistent with the WMAP1 results of Spergel et al. (2003). 

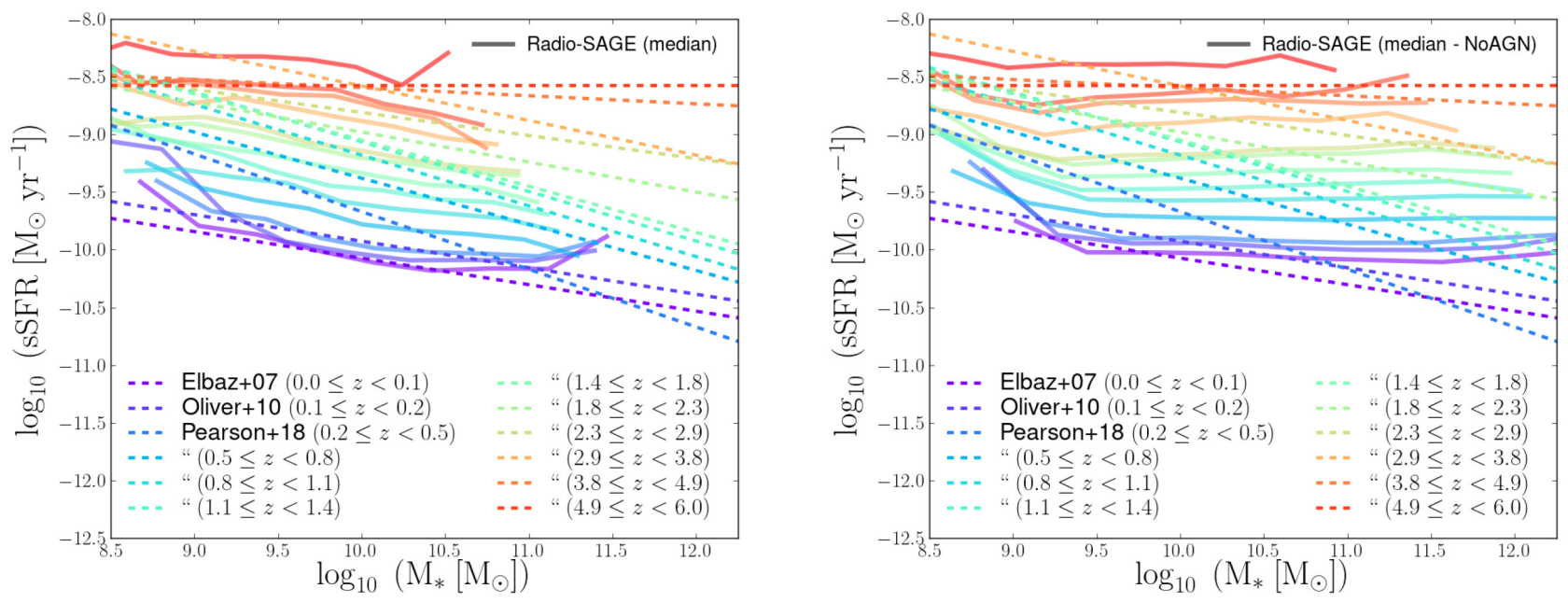

Figure 3. Left: Evolution of the sSFR - stellar mass relation. Solid lines are median binned data to the RADIO-SAGE main sequence. Dashed lines are observed trends. Right: Same but for RADIO-SAGE without AGN feedback .

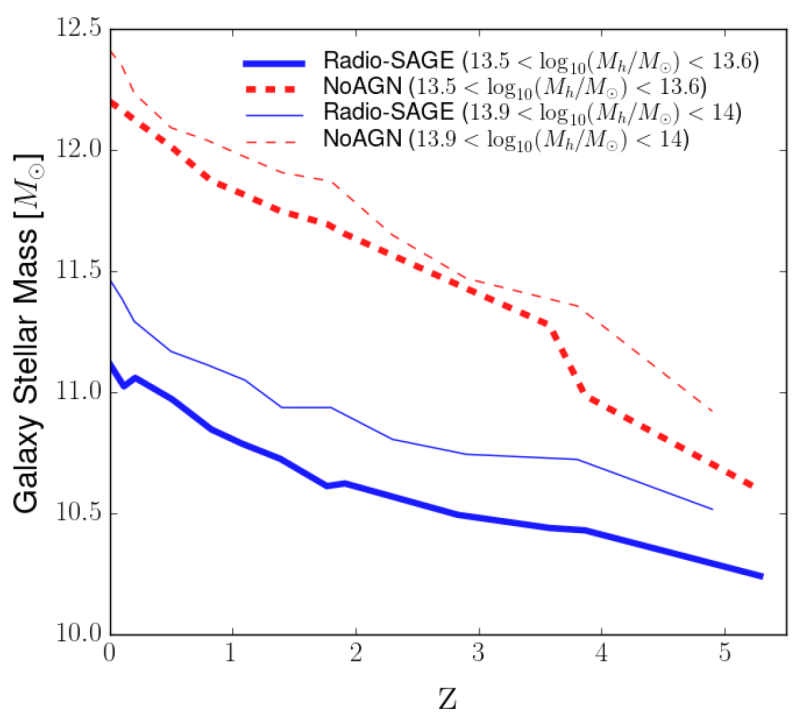

Figure 4. The median stellar mass at each redshift, for two different final halo masses in the model with (solid-line) and without (dashed-line) AGN.

model (Croton et al. 2006). The black hole accretion adopted by the ratio of accreted mass to total available cold gas mass scales with halo virial velocity (Kauffmann \& Haehnelt 2000) can be written as

$M_{\mathrm{acc}}=\frac{f_{\mathrm{BH}} m_{\mathrm{R}} M_{\mathrm{Cold}}}{1+\left(280 \mathrm{~km} \mathrm{~s}^{-1} / \mathrm{V}_{\mathrm{vir}}\right)^{2}}$,

where $m_{\mathrm{R}}=m_{1} / m_{2}$ is the mass ratio of merging galaxies (for major merger $m_{\mathrm{R}}>0.3$ ), and $f_{\mathrm{BH}}=0.015$ is the black hole growth rate. Major mergers are sufficiently energetic that the disk of the central galaxy is also destroyed and its stars added to the bulge which grows from the stellar remnants of merged satellites. Moving beyond this original accretion implementation, we built a physical model of the outflows produced as a result of gas accretion on to a black hole. The accretion rate of gas feeding the black hole (following description in Croton et al. (2006)) is approximated by the Bondi-Hoyle formula
(Bondi 1952), $\left(\dot{m}_{\mathrm{BH}} \equiv \dot{M}_{\mathrm{BH}} / \dot{M}_{\mathrm{edd}}\right)$

$\dot{m}_{\mathrm{BH}}=\frac{2.5 \pi G^{2} m_{\mathrm{BH}}^{2} \rho_{0}}{c_{s}^{3}}$,

where $m_{\mathrm{BH}}$ is the black hole mass and $\rho_{0}$ is the density of accreting hot gas around the black hole. $c_{s} \equiv V_{\text {vir }}$ and $G$ are the speed of sound in the gas and the gravitational constant, respectively. Recently, it has been argued (Hardcastle et al. 2018) that chaotic cold accretion (Gaspari et al. 2013) may provide a better description than Bondi accretion; we defer this issue to future work.

\subsection{Feedback process}

Massive dark matter haloes can contain substantial amounts of hot gas that should lead to runaway cooling at rates that are unsupported by the observations (Thoul \& Weinberg 1995; Benson et al. 2003). Our model provides an energy counterbalance to such cooling through the heating resulting from the feedback process. The effectiveness of this feedback, and at the right mass scale, is usually quantified using the galaxy stellar mass function, the shape of which is modified by both AGN and supernovae. The low-mass end is constrained by supernovae feedback processes as described in Croton et al. (2006). The high-mass end is constrained as provided by AGN feedback, and characterized by intermittent black hole accretion and resultant feedback (Shabala \& Alexander 2009).

As fully described in Raouf et al. (2017), AGN feedback is followed through different phases, which include the turning on of jets to inflate a cocoon due to black hole accretion, cessation of accretion and hence the jet which deflates the cocoon, and finally a quiescence phase of galaxies until black hole accretion begins again. The model allows cooling of gas on to the galaxy only during the quiet phase, with the duration of this time set by our prescriptions for the AGN jet on, off, and cocoon return times.

Within our model gas heating from an AGN jet acts to raise the mean temperature of the hot halo by up to $\sim 20$ per cent above the virial temperature, following the approach of Shabala \& Alexander (2009). The amount of energy available for feedback is set by the observed properties of radio AGN populations, which are mapped to physical parameters (jet power, active lifetime) using the environment-sensitive formalism of Shabala \& Godfrey (2013). In this model, we are using $\sim 35$ per cent of accretion power for 

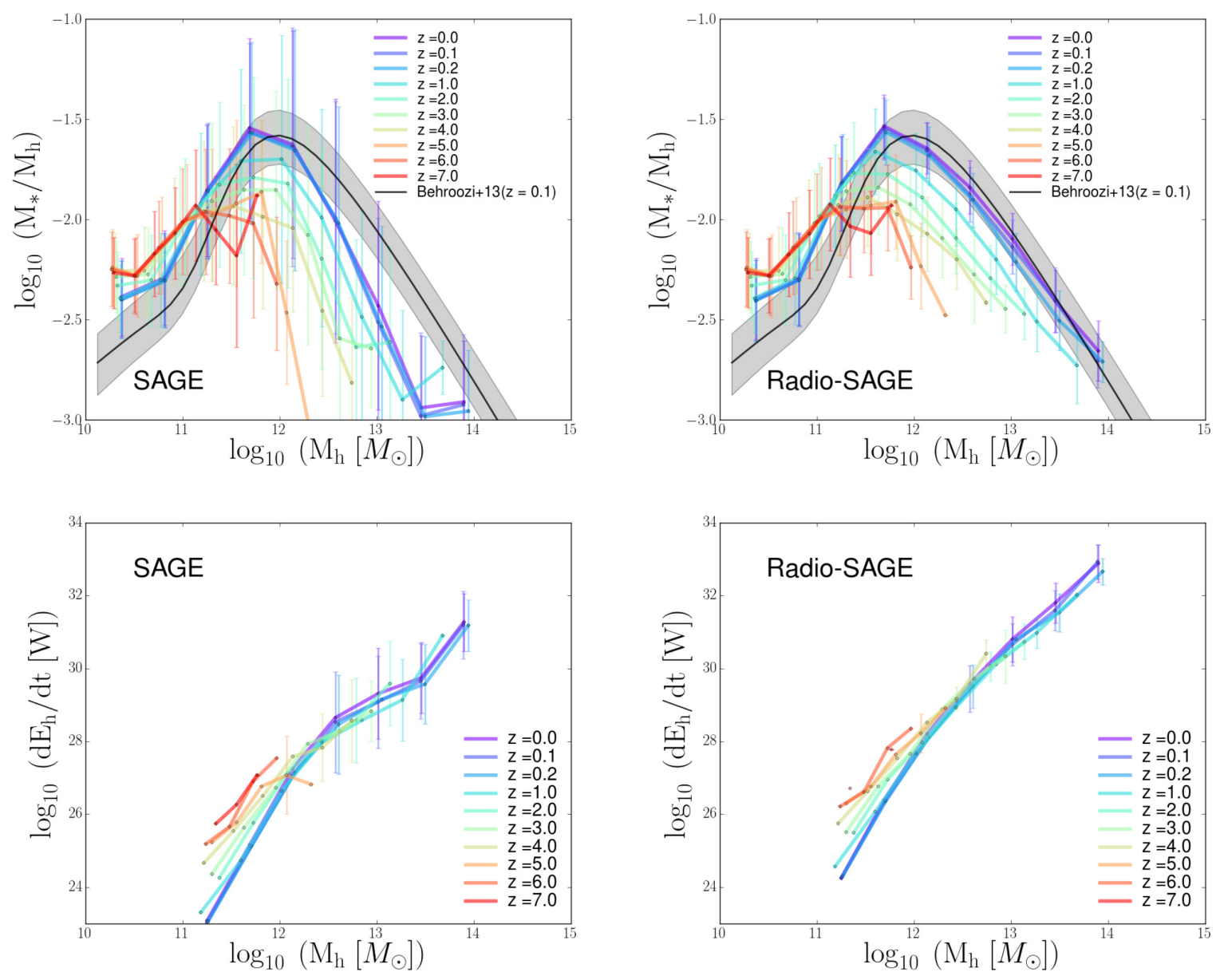

Figure 5. Top: Evolution of the derived stellar mass fractions $\left(M_{*} / M_{\mathrm{h}}\right)$ as a function of halo mass for SAGE (left) and RADIO-SAGE (right). Black lines with grey shaded uncertainties are the comparison with Behroozi et al. (2013a,b) at $z=0.1$. Bottom: Evolution of the average energy injection rate, $\mathrm{d} E_{\mathrm{h}} / \mathrm{d} t$, as a function of halo mass for RADIO-SAGE (right) and SAGE (left) in various redshift from $z=1$ to $z=7$. In each case, the lines show the mean values for central galaxies, including statistical uncertainties .

providing the necessary energy input to quench star formation and reproduce the observed local stellar mass function, the evolution of star formation density, net cooling rate temperature relation, and radio luminosity function all at the same time (Raouf et al. 2017).

\subsection{AGN outflow rate}

When the cocoon expands, some of the jet energy goes to the relativistic cocoon plasma, and some to the surrounding gas (thermal, kinetic, and gravitational components). To drive outflows, we are interested in the kinetic component. Simulations (e.g. Hardcastle \& Krause 2013, 2014; Yates et al. 2018) suggest that between 50 and 80 percent of the jet energy (depending on environment, more in low-mass systems such as galaxy groups, less in large clusters) goes to the hot intracluster medium (ICM), and between 10 and 35 percent of that value (i.e. 5-28 percent of the total jet input energy - see right panel of fig. 9 of Hardcastle \& Krause 2013) goes to the kinetic component of the ICM. The feedback power is given by

$Q_{\mathrm{jet}}=\eta \dot{m}_{\mathrm{BH}} c^{2}$,

where $c$ is the speed of light and $\eta$ is the jet efficiency which we constrain by observations to 0.35 in our model (see; Raouf et al.
2017). The outflow energy can be written by

$\dot{E}_{\mathrm{w}} \equiv \epsilon Q_{\text {jet }}$,

where $\epsilon$ is the outflow coupling efficiency where we are using the upper limit of 0.3 (Hardcastle \& Krause 2013). The outflowing gas is driven outward at a velocity $v_{\mathrm{w}}$ and mass outflow rate $\dot{M}_{\mathrm{w}}$ (Barai et al. 2014, 2016). Given the energy-conservation equation,

$\frac{1}{2} \dot{M}_{\mathrm{w}} v_{\mathrm{w}}^{2}=\dot{E}_{\mathrm{w}}$,

the outflow rate can be expressed in terms of the black hole accretion rate,

$\dot{M}_{\mathrm{w}}(\mathrm{AGN}) \equiv 2 \frac{\epsilon Q_{\mathrm{jet}}}{v_{\mathrm{w}}^{2}}\left[\frac{M_{\odot}}{\mathrm{yr}}\right]=2 \epsilon \eta\left(\frac{c}{v_{\mathrm{w}}}\right)^{2} \dot{m}_{\mathrm{BH}}$.

Here, $v_{\mathrm{w}}$ is given by

$v_{\mathrm{w}} \equiv \dot{r}_{\text {shock }}=\frac{3}{5-\beta} \frac{r_{\text {shock }}}{t_{\text {on }}}$,

where $\beta$ describes the variation of inner and outer slopes of the density profile, $t_{\mathrm{on}}$ is approximately given by Turner \& Shabala (2015)

$t_{\mathrm{on}}=120\left[\frac{m_{*}}{10^{11} \mathrm{M}_{\odot}}\right]^{0.7} \mathrm{Myr}$, 


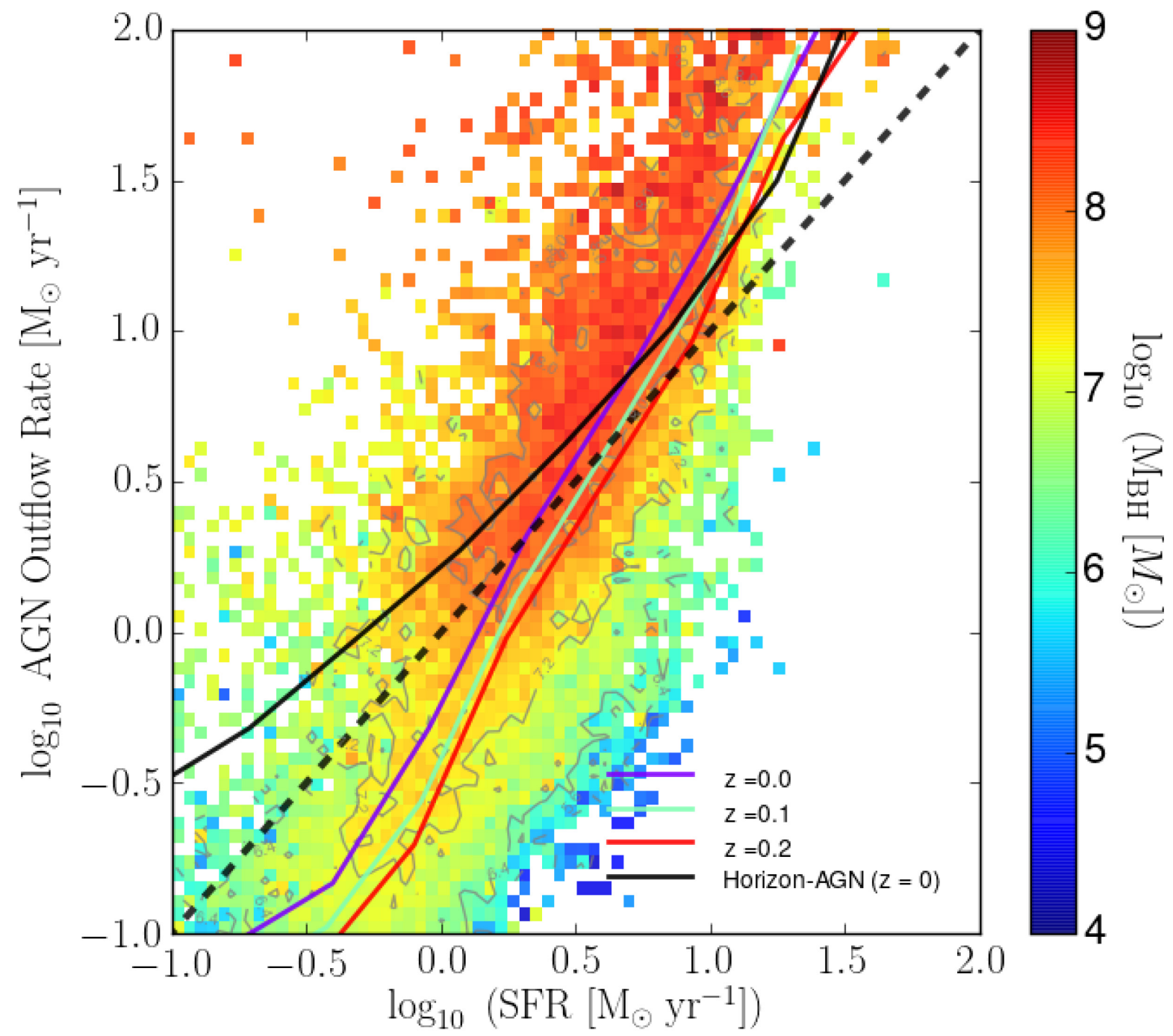

Figure 6. The local $(z<0.2)$ outflow rate versus the SFR of galaxies, colour coded by black hole mass. Coloured lines are medians in three redshift bins. The dashed line show 1:1 correspondence between the SFR and outflow rate. The black solid curve shows the median relation from Horizon-AGN hydrodynamical simulation.

and $r_{\text {shock }}$ is the maximum radius of the shocked gas describe in Raouf et al. (2017). The value of $v_{w}$ is motivated by typical AGN wind velocities seen in observations with a few 100010000 km/s (e.g. Ramirez 2008; Perna et al. 2015; Williams et al. 2016).

Here, we are arguing about the giving away of kinetic energy to the hot gas (and driving outflows in that), rather than the cold molecular/atomic gas. Note that the Kaiser \& Alexander (1997) models effectively assume momentum-conserving outflows. This is the basis for calculating $\dot{r}_{\text {shell }}$. The equation for wind outflows uses energy conservation, but it ignores thermal heating (and cooling) in the shock (on the one hand), and work done in adiabatically expanding the cocoon (on the other). Alexander (2002) showed that thermal conductivity in the swept-up shell will make a difference to the first point (i.e. whether the gas stays hot or cools rapidly). In our model, the wind estimation doesn't play an active role in the modelling; we simply use it to calculate the mass outflow rate.

\subsection{Star formation rate}

The gas does manage to cool pools in the galactic disc, and forms stars. The star formation rate can now be calculated from a Kennicutt-Schmidt-type relation (Kennicutt 1998):

$\dot{m}_{*}=\alpha_{\mathrm{SF}} \frac{\left(m_{\text {cold }}-m_{\text {crit }}\right)}{t_{\text {dyn, disk }}}$,

where $m_{\text {cold }}$ is the total mass of cold gas and $\alpha_{\mathrm{SF}}$ is the star formation efficiency $\sim 0.05$ in our model. In other words, a fraction $\alpha_{\mathrm{SF}}$ of gas above the threshold is converted into stars in a disc dynamical time $t_{\text {dyn,disk }}=r_{\text {disk }} / V_{\text {vir }}$. In this work, this star formation rate applies to both bulges and discs of galaxies.

In this paper, we focus on observed global galaxy properties and correlations, centred around star formation. Our observable figures are the star formation rate (SFR) function, the evolution of specific star formation rate (sSFR), and the star forming main sequence, shown and discussed below in Section 3. We compare our results to a 
model without AGN to show the importance of feedback at different cosmic epochs and stellar masses. We also show the improvement of our model in comparison to the original SAGE in the evolution of the stellar mass-halo mass relation. All model results assume a Universe where $h=0.73$, and when relevant, a Chabrier initial mass function (Chabrier 2003) to compare the model to the observations.

\section{MODEL CONSTRAINTS}

To calibrate this model, we use a set of observables that the output must reasonably compare with, and in a physically sensible way. Only then can we extend our analysis to explore predictions and consequences that can be compared with future observations. Note that the standard approach of matching RADIO-SAGE with observations at $z=0$ was presented in our previous paper (Raouf et al. 2017) as the calibration of our model, and here we just focus on the model SFRs in the redshift range $z=0$ to $z=7$.

\subsection{Evolution of the star formation rate}

Fig. 1 shows the SFR function of RADIO-SAGE model for the redshift ranges of $z=0-1$. In the above figure, the curves with shaded regions are the observations by Gruppioni et al. (2015) for same redshift range. The SFR function is only reasonably well modelled to $z \sim 0.5$, while the model is too efficient at quenching star formation at $z=0.5-1$. Appendix Table A 1 reports the results from the above figure.

The redshift evolution of the median specific star formation rates is shown in Fig. 2 for different stellar mass bins between $\log _{10}\left(M_{*} /\left[M_{\odot}\right]\right)=8$ and 11.5 . The trends of RADIO-SAGE sSFR in different stellar mass bins are consistent with observed data points at each redshift, in contrast to the model without AGN feedback. In our model, AGN feedback affects galaxies of all masses, albeit at different efficiencies. The results are tabulated in Appendix Table A2.

The global evolution of the correlation between the sSFR and stellar mass, the sSFR main sequence, shows in Fig. 3. The left-hand panel of Fig. 3 shows the sSFR sequence for different redshift bins in the range $z=0-6$. We compare our model with observations of Elbaz et al. (2007) for redshift range between 0.0 and 0.1, Oliver et al. (2010) for redshift range between 0.1 and 0.2, and Pearson et al. (2018) for redshift down to 6 (colour dashed-lines). The evolution of the sSFR sequence in our model is a close match to the observational trend. Further, to address in detail the question of how AGN feedback affects the star-formation main sequence, we make a similar plot for the no-AGN feedback model in the righthand panel of Fig. 3. Fig. 3 shows that radio jets are important at both low and high (e.g. $z>3$ ) redshift, where the two models predict different trends in specific star-formation main sequence. In Fig. 4, we also show the median stellar mass at each redshift, for two different narrow final halo masses for the models with and without AGN; this plot illustrates the mass scales on which AGN feedback becomes important at each redshift.

\subsection{Comparison with original SAGE}

As described in Section 2, the main differences of RADIO-SAGE with the original SAGE model are in a more sophisticated density profile and intermittent feedback from AGN (by switching off the 'radio mode' of the original SAGE model of Croton et al. (2006)). In light of these differences, it is important to consider the consistency of model predictions with recent observations. In comparison to the original SAGE, our model has better agreement with the studies by Behroozi et al. (2013a) of the relationship between stellar mass and halo mass. Sub-panels of Fig. 5 show comparison of both RADIO-SAGE and SAGE model for such a relationship. The top panels present the evolution of the derived stellar mass fractions $\left(M_{*} / M_{\mathrm{h}}\right)$ as a function of halo mass (parameters for each model are reported in Appendix Tables A5, A6). In all panels, the black lines with grey shaded error regions are the comparison with Behroozi et al. $(2013 \mathrm{a}, \mathrm{b})$. As can be seen, the trend of RADIO-SAGE have more consistency with Behroozi et al. (2013a) at the present epoch, and follows the observationally comparable trend of higher redshift evolution (see fig. 7 in Behroozi et al. 2013a) in comparison to the original SAGE. The bottom panels of the Fig. 5 show the evolution of the average energy injection rate as a function of halo mass. We calculate this energy, $\mathrm{dE}_{\mathrm{h}} / \mathrm{dt}$, by multiplying the energy injected per outburst, $Q_{\text {jet }}$, by the AGN duty cycle, $t_{\mathrm{on}} \delta$ where $\delta=0.05\left[m_{*} / 10^{11} \mathrm{M}_{\odot}\right]^{1.5}$ (Best et al. 2005), then divide by the time between redshift slices (For definition of these quantities see; Raouf et al. 2017). The figure shows where the energy is being injected, eventually resulting in different stellar masses in the two models: in RADIO-SAGE, more energy is deposited in massive $\left(>10^{13} M_{\odot}\right)$ haloes since $z=2$. Hence, our model is applying feedback in the 'right' galaxies due to the mass-dependence of the radio AGN trigger compared to the original SAGE model.

\section{RESULTS}

\subsection{Mass loading during the SFR duty cycle}

The gas content of the galaxy could be influenced directly by AGN feedback which expels the interstellar medium (ISM) out of galaxies in massive galactic winds, and/or prevents star formation by directly heating the ISM gas (Di Matteo et al. 2005; Murray, Quataert \& Thompson 2005; Springel et al. 2005; Fabian, Celotti \& Erlund 2006). While we are using a fraction of accretion power to quench star formation, massive galaxies have significant outflows depend on their black hole mass. Observational evidence supports the notion of frequent and fast outflows in massive galaxies (Tremonti, Moustakas \& Diamond-Stanic 2007) which removes large amounts of gas (Heckman et al. 2000; Veilleux, Cecil \& Bland-Hawthorn 2005; Weiner et al. 2009; Sturm et al. 2011) by using low fraction of accretion power (Moe et al. 2009; Saez et al. 2009; Dunn et al. 2010). Knowledge of the rate at which gas is blown out during the feedback process allows us to compute and analyse the massloading factor (outflow rate/SFR); the rate at which cold gas is removed from galaxies due to AGN activity.

Fig. 6 shows the outflow rate as a function of SFR of central galaxies with stellar mass over $10^{9} \mathrm{M}_{\odot}$, colour coded by black hole mass for low-redshift galaxies. There is a correlation between outflow and star formation rate across the mass scales. At lower black hole masses, the SFR is higher than the outflow rate; a similar result has been found in the HORIZON-AGN simulation (Dubois et al. 2014) that is not exclusively due to AGN feedback. Note that outflow rates in Horizon-AGN are calculated by summing over cells contained within a narrow shell of a given radius, centred on the halo. The outflow rate per cell is calculated as $\dot{\mathrm{M}}_{\mathrm{gas}}=\sum_{\mathrm{i}} \rho \Delta \mathrm{x}_{\mathrm{i}}^{3} \overline{\mathrm{v}}_{\mathrm{i}} \cdot \overline{\mathrm{r}}_{\mathrm{i}} / \omega$, where $\rho$ is the gas density, $\Delta \mathrm{x}$ is the cell size, $\overline{\mathrm{v}}_{i}$ is the gas velocity, $\overline{\mathrm{r}}_{\mathrm{i}}$ is the unit vector of the cell centre relative to the halo centre and $\omega=2 \mathrm{kpc}$ is the width of the shell. The total outflow rate for a given halo is found by summing $\dot{\mathrm{M}}_{\text {gas }}$ for all cells within the shell that have $\overline{\mathrm{v}}_{\mathrm{i}} \cdot \overline{\mathrm{r}}_{\mathrm{i}}>0$. The model has 


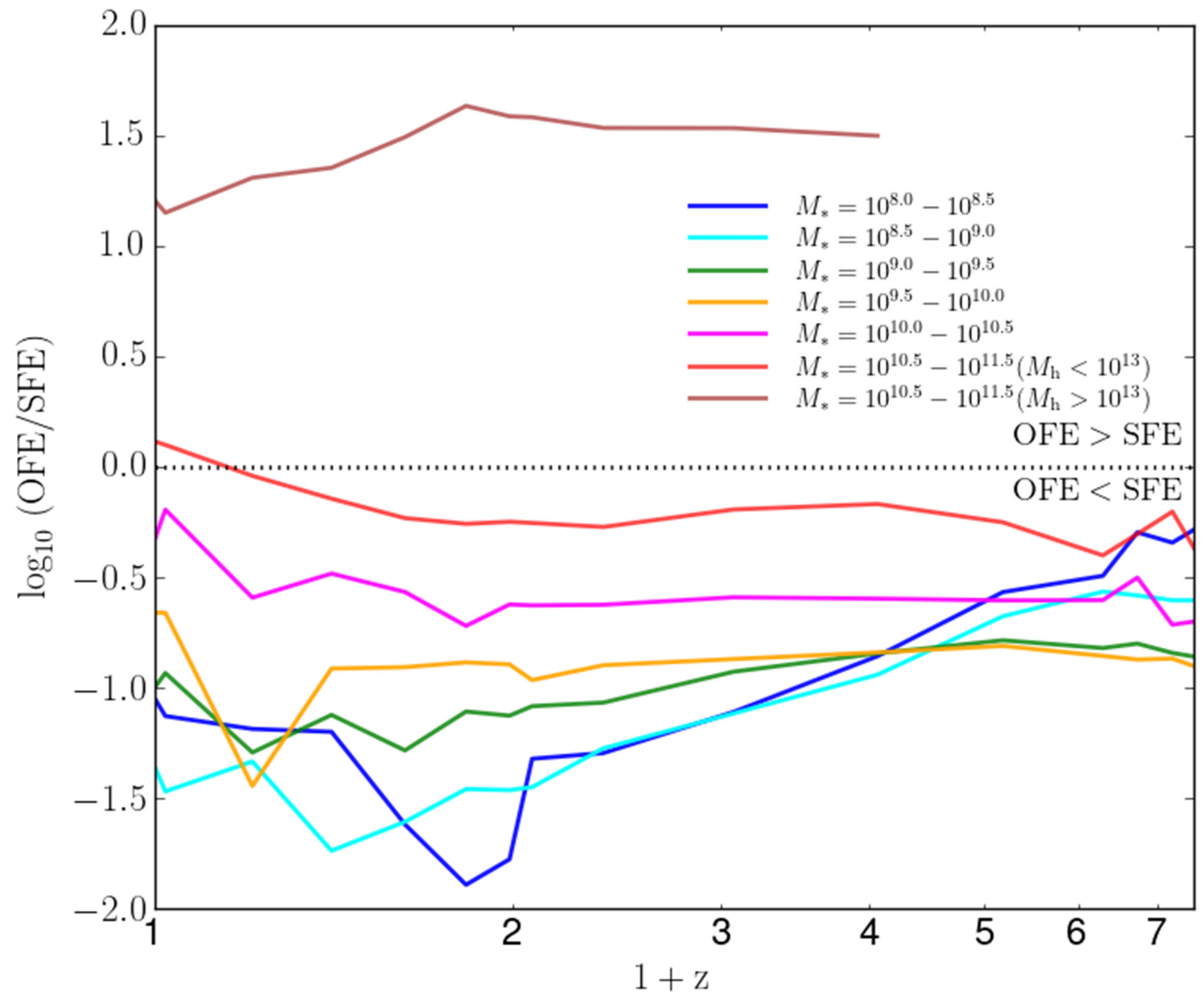

Figure 7. The redshift evolution of mass loading factor, equivalent to the ratio of outflow efficiency (OFE) to the star formation efficiency (SFE) of galaxies. The dashed line shows 1:1 correspondence between the OFE and SFE .

good agreement with the HORIZON-AGN outflow rates at the high SFR end (i.e. the high mass end, where AGN-driven outflows are dominant). At lower SFRs (and lower masses), the model for AGN winds falls short compared to the hydrodynamical simulation that includes winds from stellar feedback.

In Fig. 7, we examine the cosmological evolution of the star-formation efficiency, $\mathrm{SFE}=\mathrm{SFR} / \mathrm{M}_{\mathrm{gas}}$, and outflow efficiency, $\mathrm{OFE}=$ Outflow rate $/ \mathrm{M}_{\text {gas }}$.

In general, at a given redshift, OFE/SFE increases with stellar mass. In the galaxies with mass range of $M_{*}<10^{10.5} M_{\odot}$, the SFE consistently exceeds OFE. The most massive galaxies in the low mass clusters $\left(10^{10.5} \leq M_{*}<10^{11.5} M_{\odot}, M_{\mathrm{h}}<10^{13} M_{\odot}\right)$, the OFE approximately equals to SFE; while in the massive clusters $\left(10^{10.5} \leq M_{*}<10^{11.5} M_{\odot}, M_{\mathrm{h}}>10^{13} M_{\odot}\right)$ have higher OFE than SFEs. There is very little obvious redshift evolution in these strong mass trends. Splitting the most massive galaxies by environment (through halo mass) shows that the feedback is prevalent in the most massive galaxies clusters $\left(M_{\mathrm{h}}>10^{13} M_{\odot}\right)$, precisely the locations where the cooling catastrophe must be prevented. Note that we show the evolution of low-mass systems in the above figure just for comparison, although galaxies with stellar mass less than $10^{9} \mathrm{M}_{\odot}$ are at the resolution limit of this study.

Further, Figure A1 (appendix) shows the correlation between galaxies mass loading factor, OFE/ SFE, as a function of the black hole mass colour coded by halo mass. We also show the trend of the residuals of the best fit for the OFE/SFE $-M_{\mathrm{BH}}$ relation in different gas fraction $\left(F_{\text {gas }}\right)$, stellar mass and halo mass in the bottom panels of the above figure showing more sensitivity to the halo mass, especially in massive haloes, with respect to $F_{\text {gas }}$ and $\mathrm{M}_{*}$. Consequently, Figure A1 clearly shows that the ratio of outflow rate to SFR is mainly driven by black hole mass and virial halo mass, $M_{\text {vir }}$.

\section{DISCUSSION AND CONCLUSIONS}

In this work, we have presented the effect of black hole growth and feedback on star formation properties of galaxies using the RADIO-SAGE model. The model implements AGN feedback more realistically and self-consistently in the cooling-heating cycle which affect the process of star formation. We show a close fit to a number of key galaxy population statistics including the star formation rate function, the evolution of specific star formation rate and the specific star formation rate sequence. Expanding in the number of observables for our model, able us to make predictions for radio AGN and galaxies to be used in large-scale observing programs.

Our results in this paper are as follows. Our model is consistent with the star formation rate function up to $z=1$ and makes reasonable predictions for galaxy quenching in this redshift range. At higher redshifts, AGN feedback limits galaxy SFRs to lower rates than the model without AGN (Fig. 1). 
We find that the trend of specific star formation rate evolution with redshift is consistent with observations (Fig. 2). The AGN heating impacts the SFR over all redshift ranges of galaxies between $10^{8}$ to $10^{11.5} M_{\odot}$, and at all redshifts.

RADIO-SAGE shows better agreement with observations of the stellar mass - halo mass relation than the original SAGE model (Fig. 5). Due to the mass-dependent radio AGN fraction trigger, the RADIO-SAGE model is applying feedback in the 'right' galaxies, while SAGE is doing too much feedback in massive galaxies but not enough in low-mass ones. We suggest this could be due to the different trends of energy injection in various halo mass for the SAGE model (bottom panels of Fig. 5).

We calculate the outflow rate as a function of the star formation rate in various black hole mass bins, and find these to be broadly consistent with cosmological hydrodynamic simulations. The star formation rate is higher than the outflow rate for galaxies with a low black hole mass. We also show the quantity of star formation and outflow efficiency in different redshift and stellar mass bins of galaxies. In general, the star formation efficiency is higher than the outflow efficiency, except for the massive galaxies which host the most massive black holes and are located in massive haloes, rapidly cooling environments.

Super-massive black hole masses are strongly correlated with their host bulge stellar mass and the amount of cold gas present in a merging system plays a large part in how rapidly the black hole and bulge can grow. Future studies will focus the evolution of the $m_{\mathrm{BH}}-m_{\text {bulge }}$ relation.

\section{ACKNOWLEDGEMENTS}

We thank the referee for constructive comments and suggestions which helped to improve the paper. MR is grateful to Balzan Center for cosmological studies award and the Institut d'Astrophysique de Paris for hosting his stay during which much of this work was carried out. The Semi-Analytic Galaxy Evolution (SAGE) model that served as a basis for this work is a publicly available codebase that runs on the dark matter halo trees of a cosmological N-body simulation. It is available for download at https://github.com/darrencroton/sage. The Millennium Simulation on which the semi-analytic model was run was carried out by the Virgo Supercomputing Consortium at the Computing Centre of the Max Plank Society in Garching. It is publicly available online at http://www.mpa-garching.mpg.de/Millennium/ through the German Astrophysical Virtual Observatory. The Horizon project was supported by grant ANR-13-BS05-0005 of the French Agence Nationale de la Recherche and grant NSF PHY11- 25915 by the National Science Foundation.

\section{REFERENCES}

Alexander P., 2002, MNRAS, 335, 610

Barai P., Viel M., Murante G., Gaspari M., Borgani S., 2014, MNRAS, 437, 1456

Barai P., Murante G., Borgani S., Gaspari M., Granato G. L., Monaco P., Ragone-Figueroa C., 2016, MNRAS, 461, 1548

Behroozi P. S., Wechsler R. H., Conroy C., 2013, ApJ, 770, 57

Behroozi P. S., Wechsler R. H., Conroy C., 2013, ApJL, 762, L31

Benson A. J., Bower R. G., Frenk C. S., Lacey C. G., Baugh C. M., Cole S., 2003, ApJ, 599, 38

Best P. N., Kauffmann G., Heckman T. M. et al., 2005, MNRAS, 362, 25

Béthermin M., Daddi E., Magdis G. et al., 2015, A\&A, 573, A113

Bieri R., Dubois Y., Silk J., Mamon G. A., 2015, ApJ, 812, L36

Binney J., Tabor G., 1995, MNRAS, 276, 663
Bondi H., 1952, MNRAS, 112, 195

Booth C. M., Schaye J., 2009, MNRAS, 398, 53

Bouwens R. J., Illingworth G. D., Franx M., Ford H., 2008, ApJ, 686, 230

Bouwens R. J., Illingworth G. D., Oesch P. A. et al., 2011, ApJ, 737, 90

Bower R. G., Benson A. J., Malbon R., Helly J. C., Frenk C. S., Baugh C. M., Cole S., Lacey C. G., 2006, MNRAS, 370, 645

Cattaneo A. et al., 2009, Nature, 460, 213

Chabrier G., 2003, PASP, 115, 763

Cicone C. et al., 2014, A\&A, 562, A21

Costa T., Sijacki D., Haehnelt M. G., 2015, MNRAS, 448, L30

Crockett R. M. et al., 2012, MNRAS, 421, 1603

Croton D. J. et al., 2006, MNRAS, 365, 11

Croton D. J., Stevens A. R. H., Tonini C. et al., 2016, ApJS, 222, 22

Daddi E. et al., 2007, ApJ, 670, 156

Daddi E. et al., 2009a, ApJ, 694, 1517

Dashyan G., Silk J., Mamon G. A., Dubois Y., Hartwig T., 2018, MNRAS, 473,5698

Davis M., Efstathiou G., Frenk C. S., White S. D. M., 1985, ApJ, 292, 371

Di Matteo T., Springel V., Hernquist L. E., 2005, Nature, 433, 604

Di Matteo T., Colberg J., Springel V., Hernquist L., Sijacki D., 2008, ApJ, 676, 33

Dubois Y., Teyssier R. 2008, A\&A, 477, 79

Dubois Y., Devriendt J., Slyz A., Teyssier R., 2012, MNRAS, 420, 2662

Dubois Y., Pichon C., Welker C. et al., 2014, MNRAS, 444, 1453

Dunne L., Ivison R. J., Maddox S. et al., 2009, MNRAS, 394, 3

Dunn J. P. et al., 2010, ApJ, 709, 611

Elbaz D. et al., 2007, A\&A, 468, 33

Fabian A., 2012, ARA\&A, 50, 455

Fabian A. C., Celotti A., Erlund M. C., 2006, MNRAS, 373, L16

Farrah D. et al., 2012, ApJ, 745, 178

Ferrarese L., Merritt D., 2000, ApJ, 539, L9

Gaibler V., Khochfar S., Krause M., Silk J., 2012, MNRAS, 425, 438

Gaspari M., Ruszkowski M., Oh S. P., 2013, MNRAS, 432, 3401

Gebhardt K. et al., 2000, ApJ, 539, L13

Genzel R., Tacconi L. J., Lutz D. et al., 2015, ApJ, 800, 20

Gonzalez V. et al., 2009, preprint (arXiv:0909.3517)

González V., Bouwens R. J., Labbé I. et al., 2012, ApJ, 755, 148

Gruppioni C., Calura F., Pozzi F. et al., 2015, MNRAS, 451, 3419

Hardcastle M., 2018, Nature Astron., 2, 273

Hardcastle M. J., Krause M. G. H., 2013, MNRAS, 430, 174

Hardcastle M. J., Krause M. G. H., 2014, MNRAS, 443, 1482

Heckman T. M., Lehnert M. D., Strickland D. K., Armus L., 2000, ApJS, 129,493

Hopkins A. M., Beacom J. F., 2006, ApJ, 651, 142

Kaiser C. R., Alexander P., 1997, MNRAS, 286, 215

Kajisawa M., Ichikawa T., Yamada T. et al., 2010, ApJ, 723, 129

Kauffmann G., Haehnelt M., 2000, MNRAS, 311, 576

Kaviraj S. et al., 2015, MNRAS, 452, 774

Kennicutt R. C., 1998, ApJ, 498, 541

Khosroshahi H. G., Raouf M., Miraghaei H. et al., 2017, ApJ, 842, 81

Lee S.-K., Ferguson H. C., Somerville R. S., Wiklind T., Giavalisco M., 2010, ApJ, 725, 1644

Magdis G. E., Daddi E., Sargent M. et al., 2012b, ApJL, 758, L9

Maiolino R. et al., 2012, MNRAS, 425, 66

Makino N., Sasaki S., Suto Y., 1998, ApJ, 497, 555

McLure R. J., Dunlop J. S., de Ravel L. et al., 2011, MNRAS, 418, 2074

Moe M., Arav N., Bautista M. A., Korista K. T., 2009, ApJ, 706, 525

Murray N., Quataert E., Thompson T. A., 2005, ApJ, 618, 569

Oliver S., Frost M., Farrah D. et al., 2010, MNRAS, 405, 2279

Page M. J. et al., 2012, Nature, 485, 213

Pearson W. J., Wang L., Hurley P. D. et al., 2018, A\&A, 615, A146

Pérez-González P. G. et al., 2008, ApJ, 675, 234

Perna M. et al., 2015, A\&A, 583, A72

Quintero A. D. et al., 2004, ApJ, 602, 190

Ramirez J. M., 2008, A\&A, 489, 57

Raouf M., Khosroshahi H. G., Ponman T. J., Dariush A. A., Molaeinezhad A., Tavasoli S., 2014, MNRAS, 442, 1578

Raouf M., Khosroshahi H. G., Dariush A., 2016, ApJ, 824, 140 
Raouf M., Shabala S. S., Croton D. J., Khosroshahi H. G., Bernyk M., 2017, MNRAS, 471, 658

Raouf M., Khosroshahi H. G., Mamon G. A., Croton D., Hashemizadeh A., Dariush A. A., 2018, ApJ, 863, 40

Reddy N. A., Pettini M., Steidel C. C. et al., 2012, ApJ, 754, 25

Saez C., Chartas G., Brandt W. N., 2009, ApJ, 697, 194

Salim S. et al., 2007, ApJS, 173, 267

Santini P. et al., 2012, A\&A, 540, A109

Schaerer D., de Barros S., 2010, A\&A, 515, A73

Schawinski K. et al., 2014, MNRAS, 440, 889

Shabala S. S., Deller A., Kaviraj S., Middelberg E., Turner R. J., Ting Y. S., Allison J. R., Davis T. A., 2017, MNRAS, 464, 4706

Shabala S., Alexander P., 2009, ApJ, 699, 525

Shabala S., Godfrey L., 2013, ApJ, 769, 129

Sijacki D. et al., 2015, MNRAS, 452, 575

Sijacki D., Springel V., Di Matteo T., Hernquist L., 2007, MNRAS, 380, 877

Silk J., 2005, MNRAS, 364, 1337

Silk J., Rees M. J., 1998, A\&A, 331, L1

Sobral D. et al., 2009, MNRAS, 398, 75

Spergel D. N., Verde L., Peiris H. V. et al., 2003, ApJs, 148, 175

Springel V., White S. D. M., Tormen G., Kauffmann G. 2001, MNRAS, 328,
Springel V., White S. D. M., Jenkins A. et al., 2005, Nature, 435, 629

Stark D. P. et al., 2009, ApJ, 697, 1493

Sturm E. et al., 2011, ApJ, 733, L16

Tacconi L. J., Genzel R., Saintonge A. et al., 2018, ApJ, 853, 179

Thomas D. et al., 2005, ApJ, 621, 673

Thoul A. A., Weinberg D. H., 1995, ApJ, 442, 480

Tremonti C. A., Moustakas J., Diamond-Stanic A. M., 2007, ApJ, 663, L77

Turner R. J., Shabala S. S. 2015, ApJ, 806, 59

Veilleux S., Cecil G., Bland-Hawthorn J., 2005, ARA\&A, 43, 769

Villar V. et al., 2008, ApJ, 677, 169

Volonteri M., Dubois Y., Pichon C., Devriendt J., 2016, MNRAS, 460, 2979

Weiner B. J. et al., 2009, ApJ, 692, 187

Whitaker K. E., van Dokkum P. G., Brammer G., Franx M., 2012, ApJL, 754, L29

Williams R. J. et al., 2017, MNRAS, 467, 3399

Yates P. M., Shabala S. S., Krause M. G. H., 2018, MNRAS, 480, 5286

Zheng X. Z., Bell E. F., Papovich C. et al., 2007, ApJL, 661, L41

\section{APPENDIX : EXTRA FIGURES AND TABLES}

Table A1. Parameters of Figs 1, with units of $M_{\odot} / \mathrm{yr}$ and $\mathrm{Mpc}^{-3} \mathrm{dex}^{-1}$ for SFR and $\phi$, respectively.

\begin{tabular}{|c|c|c|c|c|c|c|}
\hline $\log _{10}(\mathrm{SFR})$ & $\phi(z=0)$ & $\phi(z=0.2)$ & $\phi(z=0.4)$ & $\phi(z=0.6)$ & $\phi(z=0.8)$ & $\phi(z=1.0)$ \\
\hline-1.01 & $1.29 \mathrm{e}-02$ & $1.60 \mathrm{e}-02$ & $1.75 \mathrm{e}-02$ & $2.07 \mathrm{e}-02$ & $2.28 \mathrm{e}-02$ & $2.26 \mathrm{e}-02$ \\
\hline$-9.11 \mathrm{e}-01$ & $1.16 \mathrm{e}-02$ & $1.51 \mathrm{e}-02$ & $1.77 \mathrm{e}-02$ & $2.12 \mathrm{e}-02$ & $2.29 \mathrm{e}-02$ & $2.40 \mathrm{e}-02$ \\
\hline$-8.09 \mathrm{e}-01$ & $1.06 \mathrm{e}-02$ & $1.35 \mathrm{e}-02$ & $1.66 \mathrm{e}-02$ & $1.93 \mathrm{e}-02$ & $2.21 \mathrm{e}-02$ & $2.41 \mathrm{e}-02$ \\
\hline$-7.07 e-01$ & $1.00 \mathrm{e}-02$ & $1.26 \mathrm{e}-02$ & $1.51 \mathrm{e}-02$ & $1.87 \mathrm{e}-02$ & $2.01 \mathrm{e}-02$ & $2.33 \mathrm{e}-02$ \\
\hline$-6.05 e-01$ & $8.84 \mathrm{e}-03$ & $1.17 \mathrm{e}-02$ & $1.38 \mathrm{e}-02$ & $1.69 \mathrm{e}-02$ & $1.99 \mathrm{e}-02$ & $2.10 \mathrm{e}-02$ \\
\hline$-5.03 e-01$ & $9.25 \mathrm{e}-03$ & $1.05 \mathrm{e}-02$ & $1.12 \mathrm{e}-02$ & $1.56 \mathrm{e}-02$ & $1.74 \mathrm{e}-02$ & $1.90 \mathrm{e}-02$ \\
\hline$-4.01 \mathrm{e}-01$ & $7.88 \mathrm{e}-03$ & $1.02 \mathrm{e}-02$ & $1.14 \mathrm{e}-02$ & $1.42 \mathrm{e}-02$ & $1.60 \mathrm{e}-02$ & $1.70 \mathrm{e}-02$ \\
\hline$-2.98 \mathrm{e}-01$ & $7.66 \mathrm{e}-03$ & $8.66 \mathrm{e}-03$ & $1.12 \mathrm{e}-02$ & $1.24 \mathrm{e}-02$ & $1.40 \mathrm{e}-02$ & $1.67 \mathrm{e}-02$ \\
\hline$-1.96 \mathrm{e}-01$ & $7.61 \mathrm{e}-03$ & $8.66 \mathrm{e}-03$ & $1.04 \mathrm{e}-02$ & $1.12 \mathrm{e}-02$ & $1.28 \mathrm{e}-02$ & $1.43 \mathrm{e}-02$ \\
\hline$-9.46 e-02$ & $8.15 \mathrm{e}-03$ & $8.70 \mathrm{e}-03$ & $9.48 \mathrm{e}-03$ & $1.05 \mathrm{e}-02$ & $1.21 \mathrm{e}-02$ & $1.31 \mathrm{e}-02$ \\
\hline $7.44 \mathrm{e}-03$ & $7.68 \mathrm{e}-03$ & $8.11 \mathrm{e}-03$ & $9.13 \mathrm{e}-03$ & $1.06 \mathrm{e}-02$ & $1.13 \mathrm{e}-02$ & $1.21 \mathrm{e}-02$ \\
\hline $1.09 \mathrm{e}-01$ & $6.94 \mathrm{e}-03$ & $7.56 \mathrm{e}-03$ & $8.90 \mathrm{e}-03$ & $9.64 \mathrm{e}-03$ & $1.11 \mathrm{e}-02$ & $1.14 \mathrm{e}-02$ \\
\hline $2.11 \mathrm{e}-01$ & $6.64 \mathrm{e}-03$ & $7.93 \mathrm{e}-03$ & $8.71 \mathrm{e}-03$ & $9.22 \mathrm{e}-03$ & $9.67 \mathrm{e}-03$ & $1.12 \mathrm{e}-02$ \\
\hline $3.13 \mathrm{e}-01$ & $6.10 \mathrm{e}-03$ & $7.23 \mathrm{e}-03$ & $8.65 e-03$ & $9.54 \mathrm{e}-03$ & $1.04 \mathrm{e}-02$ & $1.07 \mathrm{e}-02$ \\
\hline $4.15 \mathrm{e}-01$ & $5.32 \mathrm{e}-03$ & $7.02 \mathrm{e}-03$ & $7.96 \mathrm{e}-03$ & $9.25 \mathrm{e}-03$ & $9.73 e-03$ & $9.67 \mathrm{e}-03$ \\
\hline $5.18 \mathrm{e}-01$ & $5.25 \mathrm{e}-03$ & $6.40 \mathrm{e}-03$ & 7.26e-03 & $8.55 \mathrm{e}-03$ & $9.73 e-03$ & $9.97 e-03$ \\
\hline $6.20 \mathrm{e}-01$ & $3.93 \mathrm{e}-03$ & $5.38 \mathrm{e}-03$ & $6.89 \mathrm{e}-03$ & $7.99 \mathrm{e}-03$ & $8.33 \mathrm{e}-03$ & $9.83 e-03$ \\
\hline $7.22 \mathrm{e}-01$ & $3.18 \mathrm{e}-03$ & $4.30 \mathrm{e}-03$ & $5.59 \mathrm{e}-03$ & $6.80 \mathrm{e}-03$ & $8.33 e-03$ & $8.97 \mathrm{e}-03$ \\
\hline $8.24 \mathrm{e}-01$ & $2.37 \mathrm{e}-03$ & $3.44 \mathrm{e}-03$ & $5.06 \mathrm{e}-03$ & $5.75 \mathrm{e}-03$ & $7.26 \mathrm{e}-03$ & $8.12 \mathrm{e}-03$ \\
\hline $9.26 \mathrm{e}-01$ & $2.07 \mathrm{e}-03$ & $2.93 \mathrm{e}-03$ & $3.58 \mathrm{e}-03$ & $5.22 \mathrm{e}-03$ & $5.60 \mathrm{e}-03$ & $6.67 \mathrm{e}-03$ \\
\hline 1.02 & $1.38 \mathrm{e}-03$ & $2.10 \mathrm{e}-03$ & $3.20 \mathrm{e}-03$ & $3.85 \mathrm{e}-03$ & $4.89 \mathrm{e}-03$ & $5.83 e-03$ \\
\hline 1.13 & $3.82 \mathrm{e}-04$ & $1.08 \mathrm{e}-03$ & $1.78 \mathrm{e}-03$ & $2.74 \mathrm{e}-03$ & $3.05 \mathrm{e}-03$ & $4.52 \mathrm{e}-03$ \\
\hline 1.23 & $3.18 \mathrm{e}-04$ & $3.34 \mathrm{e}-04$ & $6.53 \mathrm{e}-04$ & $1.40 \mathrm{e}-03$ & $2.61 \mathrm{e}-03$ & $3.04 \mathrm{e}-03$ \\
\hline 1.33 & $1.75 \mathrm{e}-04$ & $2.70 \mathrm{e}-04$ & $4.78 \mathrm{e}-04$ & $7.96 \mathrm{e}-04$ & $1.29 \mathrm{e}-03$ & $1.68 \mathrm{e}-03$ \\
\hline 1.43 & $1.27 \mathrm{e}-04$ & $1.59 \mathrm{e}-04$ & $9.56 \mathrm{e}-05$ & $2.86 \mathrm{e}-04$ & $5.41 \mathrm{e}-04$ & $8.28 \mathrm{e}-04$ \\
\hline 1.53 & $1.11 \mathrm{e}-04$ & $1.11 \mathrm{e}-04$ & $9.56 \mathrm{e}-05$ & $1.75 \mathrm{e}-04$ & $2.70 \mathrm{e}-04$ & $3.18 \mathrm{e}-04$ \\
\hline 1.64 & $1.11 \mathrm{e}-04$ & $7.96 e-05$ & $3.18 \mathrm{e}-05$ & $1.59 \mathrm{e}-05$ & $9.56 \mathrm{e}-05$ & $1.27 \mathrm{e}-04$ \\
\hline 1.74 & $7.96 \mathrm{e}-05$ & $6.37 \mathrm{e}-05$ & 0.0 & 0.0 & $3.18 \mathrm{e}-05$ & $1.27 \mathrm{e}-04$ \\
\hline
\end{tabular}


Table A2. Parameters of Figs 2, with unit of $1 / \mathrm{yr}$ for sSFR.

\begin{tabular}{|c|c|c|c|c|c|c|}
\hline$z$ & $\begin{array}{c}\log _{10}(\mathrm{sSFR} 1) \\
\left(10^{8}-10^{8.5} M_{\odot}\right)\end{array}$ & $\begin{array}{c}\log _{10}(\mathrm{sSFR} 2) \\
\left(10^{8.5}-10^{9} M_{\odot}\right)\end{array}$ & $\begin{array}{c}\log _{10}(\mathrm{sSFR} 3) \\
\left(10^{9}-10^{9.5} M_{\odot}\right)\end{array}$ & $\begin{array}{c}\log _{10}(\mathrm{sSFR} 4) \\
\left(10^{9.5}-10^{10} M_{\odot}\right)\end{array}$ & $\begin{array}{c}\log _{10}(\mathrm{sSFR} 5) \\
\left(10^{10}-10^{10.5} M_{\odot}\right)\end{array}$ & $\begin{array}{c}\log _{10}(\text { sSFR6 }) \\
\left(10^{10.5}-10^{11.5} M_{\odot}\right)\end{array}$ \\
\hline 0 . & -9.18 & -9.51 & -9.83 & -10.00 & -10.09 & -10.12 \\
\hline 0.02 & -9.16 & -9.51 & -9.84 & -9.98 & -10.07 & -10.11 \\
\hline 0.20 & -9.16 & -9.49 & -9.75 & -9.86 & -9.94 & -10.01 \\
\hline 0.40 & -9.11 & -9.43 & -9.65 & -9.72 & -9.82 & -9.91 \\
\hline 0.62 & -9.07 & -9.38 & -9.56 & -9.59 & -9.70 & -9.80 \\
\hline 0.82 & -9.04 & -9.31 & -9.46 & -9.50 & -9.58 & -9.69 \\
\hline 0.98 & -9.01 & -9.27 & -9.38 & -9.40 & -9.50 & -9.61 \\
\hline 1.07 & -8.99 & -9.24 & -9.34 & -9.36 & -9.47 & -9.57 \\
\hline 1.38 & -8.92 & -9.14 & -9.21 & -9.23 & -9.34 & -9.47 \\
\hline 2.07 & -8.75 & -8.93 & -8.98 & -8.99 & -9.11 & -9.25 \\
\hline 3.06 & -8.50 & -8.66 & -8.71 & -8.75 & -8.88 & -9.07 \\
\hline 4.17 & -8.25 & -8.42 & -8.47 & -8.54 & -8.66 & -8.73 \\
\hline 5.28 & -8.03 & -8.22 & -8.28 & -8.37 & -8.48 & -8.55 \\
\hline 5.72 & -7.96 & -8.14 & -8.22 & -8.29 & -8.37 & - \\
\hline 6.19 & -7.90 & -8.07 & -8.17 & -8.21 & -8.48 & - \\
\hline 6.71 & -7.83 & -8.01 & -8.09 & -8.16 & -8.22 & - \\
\hline 7.27 & -7.78 & -7.95 & -8.03 & -8.06 & -8.54 & - \\
\hline
\end{tabular}



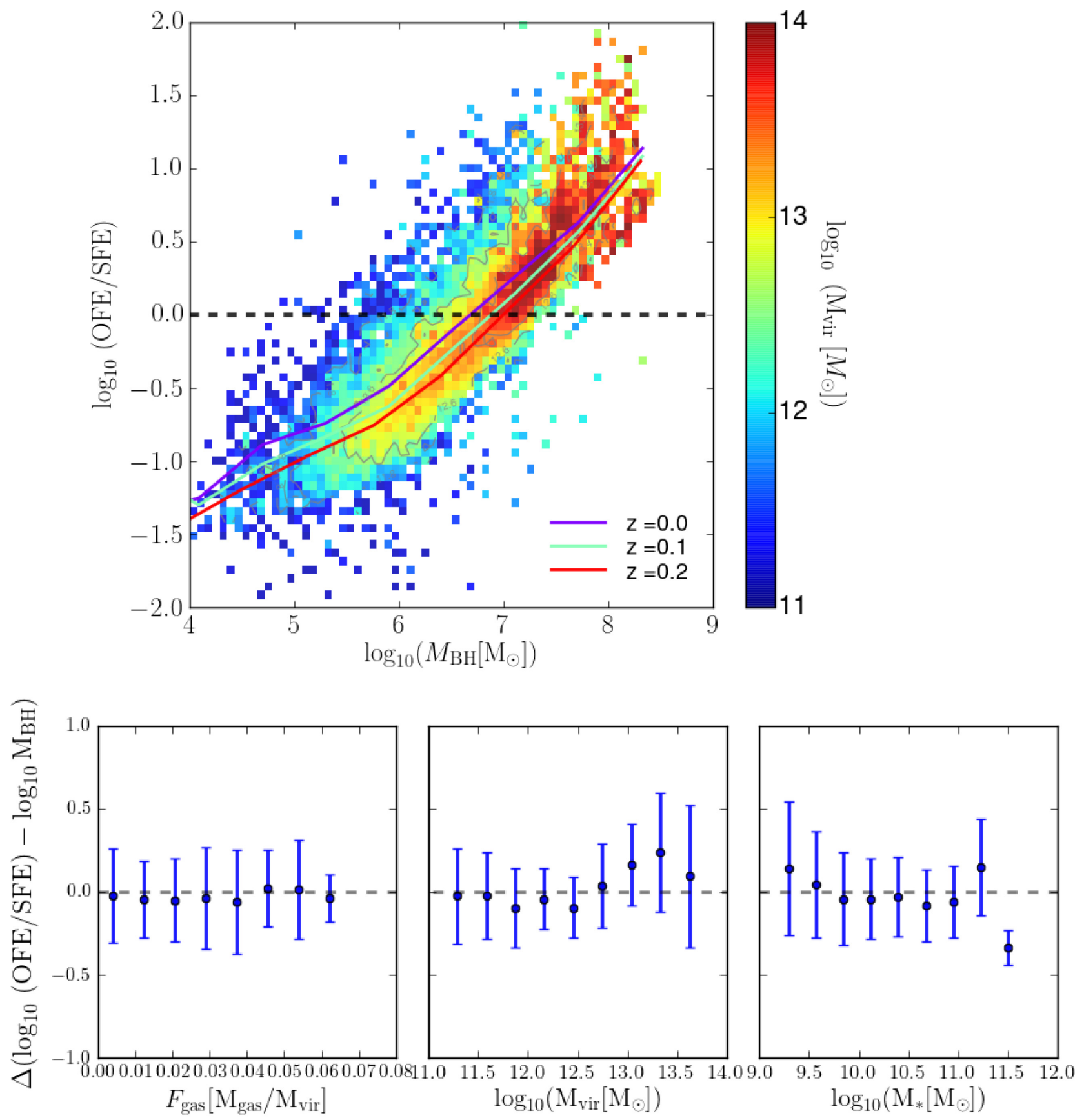

Figure A1. Top: Distribution of galaxies mass loading factor equivalent to the ratio of OFE/SFE as function of black hole mass colour code by halo mass, $M_{\text {vir }}$. Bottom panels are the residual of OFE/SFE corrected for the best-fit relation with $M_{\mathrm{BH}}$ as a function of $M_{*}$ (right), $M_{\mathrm{vir}}$ (middle), the gas fraction $M_{\mathrm{gas}} / M_{\mathrm{vir}}$ within the virial radius (left) . 
Table A3. Parameters of Figs 5 the stellar mass as a function of halo mass for $z=0,1,2,3$.

\begin{tabular}{|c|c|c|}
\hline $\log _{10}\left(M_{\mathrm{h}} / \mathrm{M}_{\odot}\right)$ & $\log _{10}\left(M_{*} / \mathrm{M}_{\odot}\right)$ & STD error (dex) \\
\hline & $z=0.0$ & \\
\hline 10.37 & 8.09 & 0.201 \\
\hline 10.81 & 8.47 & 0.299 \\
\hline 11.25 & 9.38 & 0.319 \\
\hline 11.69 & 10.10 & 0.207 \\
\hline 12.13 & 10.45 & 0.145 \\
\hline 12.57 & 10.70 & 0.126 \\
\hline 13.01 & 10.86 & 0.123 \\
\hline 13.45 & 10.97 & 0.150 \\
\hline \multirow[t]{2}{*}{13.89} & 11.19 & 0.144 \\
\hline & $z=1.0$ & \\
\hline 10.35 & 8.06 & 0.166 \\
\hline 10.77 & 8.44 & 0.297 \\
\hline 11.18 & 9.25 & 0.314 \\
\hline 11.60 & 9.89 & 0.250 \\
\hline 12.01 & 10.23 & 0.199 \\
\hline 12.43 & 10.45 & 0.178 \\
\hline 12.84 & 10.60 & 0.146 \\
\hline 13.26 & 10.69 & 0.124 \\
\hline \multirow[t]{2}{*}{13.67} & 10.90 & 0.057 \\
\hline & $z=2.0$ & \\
\hline 10.32 & 8.05 & 0.161 \\
\hline 10.67 & 8.35 & 0.273 \\
\hline 11.02 & 9.00 & 0.306 \\
\hline 11.37 & 9.57 & 0.284 \\
\hline 11.73 & 9.93 & 0.226 \\
\hline 12.08 & 10.16 & 0.197 \\
\hline 12.43 & 10.29 & 0.168 \\
\hline 12.78 & 10.45 & 0.127 \\
\hline \multirow[t]{2}{*}{13.13} & 10.58 & 0.128 \\
\hline & $z=3.0$ & \\
\hline 10.31 & 8.08 & 0.168 \\
\hline 10.64 & 8.36 & 0.286 \\
\hline 10.97 & 8.92 & 0.296 \\
\hline 11.30 & 9.40 & 0.284 \\
\hline 11.62 & 9.76 & 0.248 \\
\hline 11.95 & 9.99 & 0.204 \\
\hline 12.28 & 10.18 & 0.193 \\
\hline 12.61 & 10.25 & 0.213 \\
\hline 12.94 & 10.42 & 0.196 \\
\hline
\end{tabular}

Table A4. Parameters of Figs 5 the stellar mass as a function of halo mass for $z=4,5,6,7$.

\begin{tabular}{|c|c|c|}
\hline $\log _{10}\left(M_{\mathrm{h}} / \mathrm{M}_{\odot}\right)$ & $\begin{array}{c}\log _{10}\left(M_{*} / \mathrm{M}_{\odot}\right) \\
z=4.0\end{array}$ & STD error (dex) \\
\hline 10.30 & 8.10 & 0.179 \\
\hline 10.60 & 8.34 & 0.247 \\
\hline 10.91 & 8.82 & 0.287 \\
\hline 11.21 & 9.26 & 0.273 \\
\hline 11.52 & 9.59 & 0.257 \\
\hline 11.82 & 9.80 & 0.247 \\
\hline 12.13 & 10.05 & 0.185 \\
\hline 12.43 & 10.14 & 0.115 \\
\hline \multirow[t]{2}{*}{12.74} & 10.24 & 0.075 \\
\hline & $z=5.0$ & \\
\hline 10.27 & 8.08 & 0.181 \\
\hline 10.53 & 8.24 & 0.226 \\
\hline 10.79 & 8.64 & 0.262 \\
\hline 11.04 & 9.03 & 0.271 \\
\hline 11.30 & 9.32 & 0.261 \\
\hline 11.55 & 9.60 & 0.243 \\
\hline 11.81 & 9.87 & 0.228 \\
\hline 12.06 & 9.81 & 0.206 \\
\hline \multirow[t]{2}{*}{12.32} & 9.77 & 0. \\
\hline & $z=6.0$ & \\
\hline 10.27 & 8.06 & 0.178 \\
\hline 10.51 & 8.22 & 0.209 \\
\hline 10.75 & 8.58 & 0.253 \\
\hline 10.99 & 8.94 & 0.254 \\
\hline 11.24 & 9.26 & 0.255 \\
\hline 11.48 & 9.52 & 0.226 \\
\hline 11.72 & 9.73 & 0.223 \\
\hline \multirow[t]{2}{*}{11.96} & 9.73 & 0.122 \\
\hline & $z=7.0$ & \\
\hline 10.29 & 8.08 & 0.162 \\
\hline 10.50 & 8.22 & 0.194 \\
\hline 10.71 & 8.53 & 0.227 \\
\hline 10.92 & 8.81 & 0.229 \\
\hline 11.13 & 9.18 & 0.237 \\
\hline 11.34 & 9.23 & 0.257 \\
\hline 11.55 & 9.38 & 0.224 \\
\hline 11.76 & 9.73 & 0. \\
\hline
\end{tabular}


Table A5. Parameters of Figs 5 the derived stellar mass fractions $\left(M_{*} / M_{h}\right)$ as a function of halo mass for $z=0,1,2,3$.

\begin{tabular}{|c|c|c|}
\hline $\log _{10}\left(M_{\mathrm{h}} / \mathrm{M}_{\odot}\right)$ & $\begin{array}{c}\log _{10}\left(M_{*} / M_{h}\right) \\
z=0.0\end{array}$ & STD error (dex) \\
\hline 10.37 & -2.39 & 0.203 \\
\hline 10.81 & -2.29 & 0.231 \\
\hline 11.25 & -1.82 & 0.235 \\
\hline 11.69 & -1.53 & 0.158 \\
\hline 12.13 & -1.64 & 0.126 \\
\hline 12.57 & -1.83 & 0.132 \\
\hline 13.01 & -2.09 & 0.129 \\
\hline 13.45 & -2.40 & 0.154 \\
\hline \multirow[t]{2}{*}{13.89} & -2.65 & 0.149 \\
\hline & $z=1.0$ & \\
\hline 10.35 & -2.39 & 0.169 \\
\hline 10.77 & -2.29 & 0.229 \\
\hline 11.18 & -1.88 & 0.254 \\
\hline 11.60 & -1.66 & 0.214 \\
\hline 12.01 & -1.75 & 0.189 \\
\hline 12.43 & -1.94 & 0.181 \\
\hline 12.84 & -2.20 & 0.172 \\
\hline 13.26 & -2.51 & 0.148 \\
\hline \multirow[t]{2}{*}{13.67} & -2.72 & 0.190 \\
\hline & $z=2.0$ & \\
\hline 10.32 & -2.33 & 0.169 \\
\hline 10.67 & -2.30 & 0.226 \\
\hline 11.02 & -1.98 & 0.263 \\
\hline 11.37 & -1.76 & 0.253 \\
\hline 11.73 & -1.77 & 0.214 \\
\hline 12.08 & -1.89 & 0.192 \\
\hline 12.43 & -2.09 & 0.176 \\
\hline 12.78 & -2.29 & 0.165 \\
\hline \multirow[t]{2}{*}{13.13} & -2.47 & 0.128 \\
\hline & $z=3.0$ & \\
\hline 10.31 & -2.28 & 0.177 \\
\hline 10.64 & -2.27 & 0.230 \\
\hline 10.97 & -2.01 & 0.263 \\
\hline 11.30 & -1.85 & 0.263 \\
\hline 11.62 & -1.83 & 0.236 \\
\hline 11.95 & -1.92 & 0.207 \\
\hline 12.28 & -2.09 & 0.196 \\
\hline 12.61 & -2.27 & 0.241 \\
\hline 12.94 & -2.44 & 0.119 \\
\hline
\end{tabular}

Table A6. Parameters of Figs 5 the derived stellar mass fractions $\left(M_{*} / M_{h}\right)$ as a function of halo mass for $z=4,5,6,7$.

\begin{tabular}{|c|c|c|}
\hline $\log _{10}\left(M_{\mathrm{h}} / \mathrm{M}_{\odot}\right)$ & $\begin{array}{c}\log _{10}\left(M_{*} / M_{h}\right) \\
z=4.0\end{array}$ & STD error (dex) \\
\hline 10.30 & -2.26 & 0.189 \\
\hline 10.60 & -2.25 & 0.218 \\
\hline 10.91 & -2.05 & 0.255 \\
\hline 11.21 & -1.92 & 0.253 \\
\hline 11.52 & -1.88 & 0.242 \\
\hline 11.82 & -1.97 & 0.233 \\
\hline 12.13 & -2.07 & 0.184 \\
\hline 12.43 & -2.19 & 0.149 \\
\hline \multirow[t]{2}{*}{12.74} & -2.41 & 0.012 \\
\hline & $z=5.0$ & \\
\hline 10.27 & -2.24 & 0.191 \\
\hline 10.53 & -2.28 & 0.206 \\
\hline 10.79 & -2.12 & 0.238 \\
\hline 11.04 & -1.99 & 0.256 \\
\hline 11.30 & -1.94 & 0.244 \\
\hline 11.55 & -1.93 & 0.241 \\
\hline 11.81 & -1.91 & 0.234 \\
\hline 12.06 & -2.23 & 0.213 \\
\hline \multirow[t]{2}{*}{12.32} & -2.47 & 0. \\
\hline & $z=6.0$ & \\
\hline 10.27 & -2.24 & 0.185 \\
\hline 10.51 & -2.28 & 0.192 \\
\hline 10.75 & -2.14 & 0.231 \\
\hline 10.99 & -2.01 & 0.241 \\
\hline 11.24 & -1.93 & 0.241 \\
\hline 11.48 & -1.94 & 0.219 \\
\hline 11.72 & -1.94 & 0.213 \\
\hline \multirow[t]{2}{*}{11.96} & -2.23 & 0.158 \\
\hline & $z=7.0$ & \\
\hline 10.29 & -2.26 & 0.174 \\
\hline 10.50 & -2.28 & 0.184 \\
\hline 10.71 & -2.17 & 0.209 \\
\hline 10.92 & -2.07 & 0.220 \\
\hline 11.13 & -1.92 & 0.231 \\
\hline 11.34 & -2.03 & 0.253 \\
\hline 11.55 & -2.06 & 0.208 \\
\hline 11.76 & -1.93 & 0. \\
\hline
\end{tabular}

This paper has been typeset from a $\mathrm{T}_{\mathrm{E}} \mathrm{X} / \mathrm{LAT}_{\mathrm{E}} \mathrm{X}$ file prepared by the author. 